\title{
Investigation of the assessment of low degree $(<50 \%)$ renal artery stenosis based on velocity flow profile analysis using Doppler ultrasound: An in-vitro study
}

\author{
Andrew Malone \\ Technological University Dublin, andrew.malone@tudublin.ie \\ Deepa Chari \\ Florida International University \\ Sean Cournane \\ St Vincent's University Hospital
}

See next page for additional authors

Follow this and additional works at: https://arrow.tudublin.ie/scschphyart

Part of the Medicine and Health Sciences Commons, and the Physics Commons

\section{Recommended Citation \\ Malone A, Chari D, Cournane S, Naydenova I, Fagan A, Browne J. Investigation of the assessment of low degree $(<50 \%)$ renal artery stenosis based on velocity flow profile analysis using Doppler ultrasound: An in-vitro study. Phys Med. 2019 Sep;65:209-218. doi: 10.1016/j.ejmp.2019.08.016. Epub 2019 Sep 10. \\ PMID: 31518907.}

This Article is brought to you for free and open access by the School of Physics \& Clinical \& Optometric Science at ARROW@TU Dublin. It has been accepted for inclusion in Articles by an authorized administrator of ARROW@TU Dublin. For more information, please contact arrow.admin@tudublin.ie, aisling.coyne@tudublin.ie, gerard.connolly@tudublin.ie.

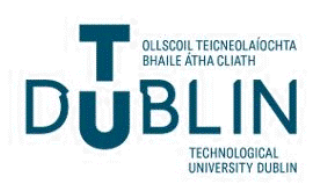


Authors

Andrew Malone, Deepa Chari, Sean Cournane, Izabela Naydenova, Andrew Fagan, and Jacinta Browne 


\section{Investigation of the assessment of low degree $(<50 \%)$ renal artery stenosis based on velocity flow profile analysis using Doppler ultrasound: An in-vitro study}

Andrew Malone ${ }^{a, *}$, Deepa Chari ${ }^{b}$, Sean Cournane ${ }^{c}$, Izabela Naydenova ${ }^{a}$, Andrew Fagan $^{d}$, and Jacinta Browne ${ }^{a, d}$

a School of Physics and Clinical \& Optometric Sciences, College of Science and Health, Technological University Dublin, Dublin, Ireland b STEM Transformation Institute, Florida International University, Miami, USA c St Vincent's University Hospital, Dublin, Ireland d Department of Radiology, Mayo Clinic, Rochester, MN, USA

\section{Abstract}

Purpose: Renal arterial stenosis can lead to disrupted renal function due to reduced blood flow to the kidneys and is largely thought to be caused by atherosclerosis. Current diagnostic strategies for renal arterial stenosis rely on detecting large degree stenoses (>50\%). This study aimed to test the viability of using Doppler ultrasound to assess velocity profiles to detect the presence of low degree $(<50 \%)$ stenoses.

Methods: A series of anatomically realistic renal artery flow phantoms were constructed exhibiting a range of low degree stenoses (symmetric and asymmetric). The behaviour of fluid flow in the phantoms was examined using Doppler ultrasound and analysed to calculate the clinical biomarker, wall shear stress.

Results: $A$ number of fluid behaviours were observed in relation to stenosis degree: asymmetric stenoses tended to result in a skewing of peak velocities away from the centre of the vessel towards the outer wall, the magnitude of increase in velocity was observed to correlate with stenosis degree, and the wall shear stress curves observed large peaks in the presence of even the lowest degree stenosis (20\%).

Conclusions: Doppler ultrasound could potentially be utilised to diagnose low degree stenoses in a clinical setting. Doppler ultrasound in conjunction with wall shear stress analysis in particular has significant potential in the diagnosis of renal artery stenosis.

Keywords: Doppler ultrasound, wall shear stress, renal artery stenosis, flow phantom

\section{Introduction}

Renal artery stenosis (RAS) is a narrowing of the major arteries that supply blood to the kidneys. One of the main causes of RAS is atherosclerosis which is characterised as the deposition of atherosclerotic plaque on the walls of the artery. An estimated $90 \%$ of RAS is due to atherosclerosis [1]. Atherosclerotic RAS disturbs the typical behaviour of blood flow, reduces renal perfusion pressure, and leads to renovascular hypertension. Renovascular hypertension is a relatively rare form of secondary hypertension, presenting in $1-5 \%$ of hypertension cases, however it is of clinical interest due to it being potentially curable through angioplasty or surgery [2]. There is also evidence to suggest that the presence of atherosclerotic RAS is indicative of additional atherosclerotic plaque 
elsewhere in the body such as the carotid artery and coronary artery; which emphasises the importance of RAS diagnosis techniques [3]. Furthermore, RAS is a progressive disease which can lead to significant health complications if left untreated such as: progressive renal insufficiency, myocardial infarction, stroke, and death [4]. Additionally, the surgical treatments commonly used for treatment in advanced cases of RAS are associated with complications such as trauma and bleeding. There is, therefore, a need for a diagnostic tool to provide earlier diagnosis of RAS, so that less invasive drug treatments can be explored which pose a much lower risk of complication to patients.

Several methods are used for the diagnosis of RAS including magnetic resonance angiography (MRA), computed tomography angiography (CTA), and Doppler ultrasound (US). MRA and CTA procedures carry a risk to the patient due to nephrotoxicity as a result of the contrast agents used, while the techniques may lead to the overestimation of the stenosis degree in addition [5]. Due to its high degree of sensitivity in the assessment of RAS, digital subtraction angiography (DSA) has been utilised as the "gold standard" for the diagnosis of RAS, however it is an ionising and invasive technique and so is now only utilised for assessment of below-the-knee arterial disease and in cases where there is disagreement between non-invasive diagnostic techniques [5].

It long been understood that the blood velocity flow profile has a significant impact on the development of RAS [6]. A clinical biomarker related to the development of RAS is wall shear stress (WSS), which is defined as the product of the dynamic blood viscosity and the gradient of the blood velocity profile evaluated at the vessel wall. In the 1970s, it was theorised that a high WSS would cause damage to the endothelium resulting in the formation of atherosclerotic lesions, however Caro et al. proposed that results in vivo indicated that it was low WSS values which coincided with areas of increased lesion formation [6]. This was later confirmed experimentally in the 1980s and early 1990s through a number of studies, also, demonstrating that the measurement of WSS was of critical importance in the early diagnosis of RAS [7]-[10]. Malek et al. provides a detailed analysis of the historical evolution of understand of the role of WSS in atherosclerosis and, thus, provides a clear incentive for developing a more thorough understanding of blood velocity flow profiles in the diagnosis of RAS [11].

Consequently, there is clinical interest in improving the assessment capabilities of the blood velocity profile for non-invasive diagnostic modalities. Of these modalities, Doppler ultrasound presents as the least expensive option, in addition to being non-ionising, real-time and widely available. Clinical studies involving Doppler ultrasound in the diagnosis of RAS compared to DSA have reported sensitivity of between $67 \%$ to $97 \%$ and specificity between $54 \%$ to $92 \%$, for the former compared to a sensitivity between $94 \%$ to $100 \%$ and a specificity of $65 \%$ to $97 \%$ for the latter [12]-[16]. Despite the advantages of Doppler ultrasound, it has several limitations compared to DSA, including: the dependence on the skill of the operator, limited ability to measure high velocities at depth without aliasing, and reduced measurement sensitivity for difficult patients, such as obese patients or where there is a significant presence of gas [5].

In order to explore the role of Doppler ultrasound in the early diagnosis of RAS, it is necessary to develop a standard test bed for evaluating the effectiveness of the approach. Previous investigations for developing novel Doppler ultrasound techniques for characterising disease have been initially carried out on tissue mimicking test objects and the methods used for the production of these phantoms have been varied and their usefulness to this work is uncertain [17]-[26]. As such, there is a need for the development of anatomically realistic flow phantoms which can be easily and reproducibly manufactured. The phantoms should incorporate features of disease progression, such as vessel stenosis, and be suitable for imaging with different imaging modalities producing distortion-less images. 
The aim of this study was to investigate whether Doppler ultrasound could be used to determine velocity profiles and local changes in haemodynamic profiles in order to detect the presence of a $<$ $50 \%$ stenosis. This was achieved through an in-vitro study which involved the use of wall-less, anatomically realistic renal artery flow phantoms presenting varying degrees of symmetrical (normal, $30 \%, 50 \%$ ) and asymmetric $(25 \%, 35 \%, 45 \%)$ non haemodynamically significant stenosis. The effect of the degree of stenosis on blood flow velocity profiles in the presence of physiological flow was investigated.

\section{Materials and Methods}

\section{Model Manufacture}

The anatomically realistic renal artery models were generated from 3D computer model of a healthy renal artery from a 3D CT clinical dataset and were fabricated using the methodology previously described by King et al. (2010) [20]. The patient data used to produce renal artery computer model had a lumen diameter of $7 \mathrm{~mm}$ which falls on the high end of typical renal artery diameters of $3.0-$ $6.8 \mathrm{~mm}$ reported by Aytac et al. [27]. To summarise, the CT dataset was used to rapid prototype a master renal artery model (Model: Z printer 310 Plus 3D, Zcorporation, MA, USA) which was used to produce a master silicone negative mould from which renal artery cores could be produced. Using this silicone mould low melting point alloy (melting point: $47^{\circ} \mathrm{C}, \mathrm{MCP} 47$, Mining and Chemical Products Ltd., Northamptonshire, UK) was used to manufacture cores of the renal artery geometry. The degree of stenosis could be controlled through the use of inserts incorporated in the master silicone mould which narrowed the model width at the position of the stenosis. The renal artery metal cores of $30 \%$ and $50 \%$ concentric stenosis and of $25 \%, 35 \%$ and $45 \%$ eccentric stenosis are presented in Figure 1(a) and 1(b), respectively.

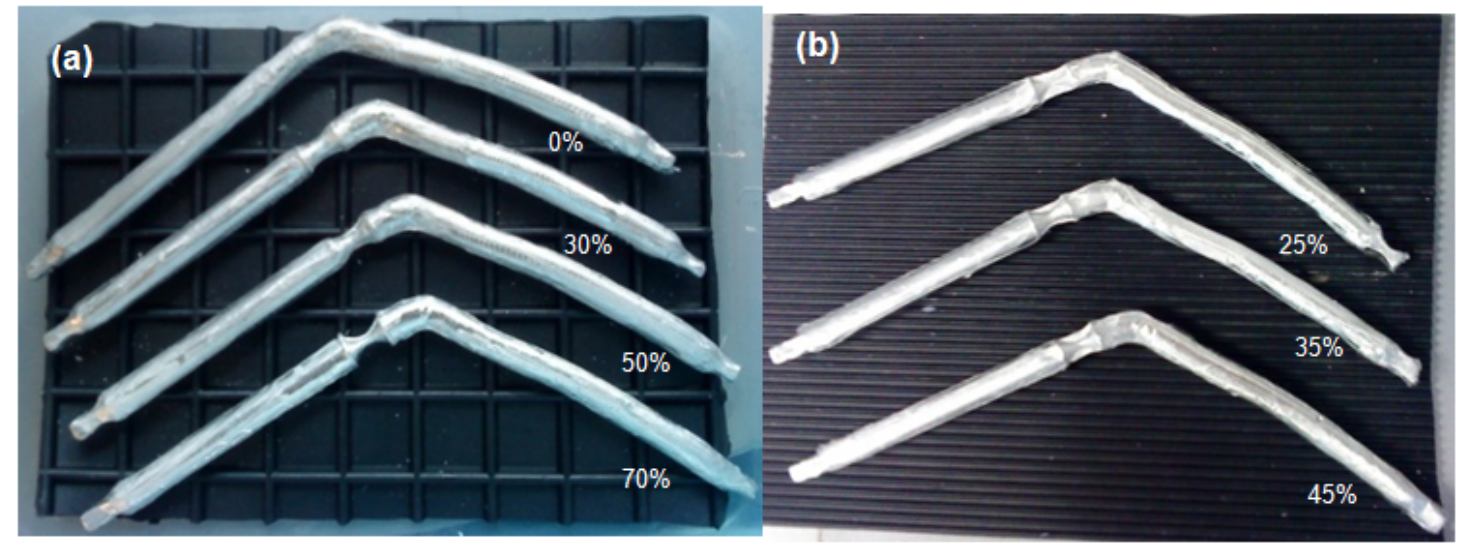

Figure 1: Renal artery metal cores (a) normal model and with $30 \%, 50 \%$ and $70 \%$ concentric stenosis and (b) with eccentric $25 \%, 35 \%$ and $45 \%$ stenosis.

\section{Phantom Construction}

The phantom containers dimensions were $230 \mathrm{~mm} \times 135 \mathrm{~mm} \times 103 \mathrm{~mm}(\mathrm{~L} \times \mathrm{W} \times \mathrm{H})$ and a wall thickness of $2.6 \mathrm{~mm}$. 2 plastic $7.5 \mathrm{~mm}$ barbed connecters (Product number 06360-90, Cole-Parmer, Walden, UK) were secured to the walls of the phantom container to act as inlet and outlet ports. 7.9 mm Nalgene ${ }^{\circledR}$ PVC plastic tubing (Product number 228-0607, VWR International, Dublin, Ireland) was attached to the connectors to allow the metal core to be secured between them. Reticulated foam (30 pores per inch, Foam Techniques, Northamptonshire, UK) (60 mm x $60 \mathrm{~mm} \times 10 \mathrm{~mm}$ ) was fixed using Araldite ${ }^{\mathrm{TM}}$ adhesive applied around the sides of the phantom container, the connectors and tubing to help seal the phantom. Before securing the renal artery metal core in place, a single layer 
of clingfilm (thickness $0.1 \mathrm{~mm}$ ) was wrapped around it. This was to overcome the problem of residual metal encountered by King et al. (2010) [20]. At each end of the metal core an extra length of clingfilm was passed out through the connectors. The renal artery metal core was secured between the two plastic tubes. An agar-based TMM was used [28]. This TMM is very well characterised and has good reproducibility and our results were found to agree with those found by other groups [28]-[31]. The TMM was characterised and found to have a speed of sound of $1541 \pm 3$ $\mathrm{m} \mathrm{s}^{-1}$ and attenuation coefficient of $0.52 \pm 0.03 \mathrm{~dB} \mathrm{~cm}^{-1} \mathrm{MHz}^{-1}$. The TMM was allowed to cool to $42^{\circ} \mathrm{C}$ (stirring continuously), and then poured into the phantom container until $4.5 \mathrm{~cm}$ thickness of TMM covered the renal artery metal core, this represented the typical depth of renal arteries in-vivo. When the TMM was set, the phantom was placed in a temperature-controlled water bath $\left(50^{\circ} \mathrm{C} \leq \mathrm{T}\right.$ $\leq 60^{\circ} \mathrm{C}$ ) for approximately 4 hours to allow the renal artery metal core (melting point $47^{\circ} \mathrm{C}$ ) to melt. No agar TMM was directly exposed to water in the bath as the phantom was sealed with a lid and clingfilm and metal core remained in the lumen until the phantom was removed from the bath. The clingfilm containing the molten metal was carefully removed; resulting in a realistic renal artery lumen with no residual metal remaining. Seven renal artery flow phantoms representing the range of stenosis outlined above were constructed following the above procedure using the corresponding metal model and insert for the respective stenosis size. The constructed renal artery flow phantoms were imaged using B- mode ultrasound with a linear array transducer $(5-13 \mathrm{MHz})$ of a Siemens Antares Ultrasound system. The lumen was observed in the B-mode images to determine if the lumen was free from metal residue. If necessary, the lumen was cleaned carefully using pipe cleaners with care being taken not to disturb the set TMM. To ensure long term stability of the phantoms, when not in use, they were stored in air-tight containers at room temperature with the scanning wells and lumina of the phantoms filled with a solution of $87.7 \%$ water, $11.8 \%$ glycerol, and $0.5 \%$ Benzalkonium chloride (10\% concentration) similar to the water, glycerol, and Benzalkonium chloride concentration in the TMM. This was to prevent the phantoms from drying out, to prevent bacterial growth and also prevent a change in speed of sound due to loss of glycerol through diffusion.

\section{Geometric Accuracy of the Renal Artery Flow Phantoms}

The geometric accuracy of the renal artery flow phantom development was determined by comparing the renal artery diameters measured at two different stages of development: a) at the metal core/model stage and b) the final renal lumen diameter in the flow phantom. The renal artery metal model diameter was measured at three sites for all metal models using a Vernier callipers (accuracy $\pm 0.01 \mathrm{~mm}$ ). The renal lumen diameter was measured at three different sites in the renal flow phantoms from B-mode images of the renal flow phantoms using ultrasound system' $s$ electronic callipers (accuracy $\pm 0.1 \mathrm{~mm}$ ). The B-mode images were acquired using a linear transducer (5-13 MHz) on a Siemens Antares system and three observations were recorded for each measurement site. To assess the geometric accuracy of the flow phantom development, the metal model measurement and the renal lumen diameters measured in the B-mode images were statistically compared using a paired t-test. Ten observations for the diameter measured at the above-mentioned stages were recorded and using a paired t-test the corresponding $p$ values were determined.

\section{Ultrasound Flow Data}

The flow phantoms were connected to an in-house built flow circuit system which provided the invitro flow (Figure 2a). The flow circuit consisted of a magnetically driven cavity style pump head (Cole Parmer, Walden, UK) used to pump the Blood Mimicking Fluid [28] through the flow circuit coupled to a direct current servo motor (McLennan Servo Suppliers Ltd., Surrey, UK). The motor was 
driven by a servo amplifier (Aerotech Ltd, Berkshire, UK), multifunctional I/O board (National Instruments, Austin, TX, USA) and a computer controller program developed in LABVIEW (National Instruments, Austin, TX, USA) was used to output the required velocities (calibrated using timed weight collection). The BMF was degassed using a sonicator and was pumped through the phantoms and flow circuit (Figure 2b) for 1 hour prior to measurements to ensure no air bubbles remained. An inlet length of $100 \mathrm{~cm}$ was used to ensure that laminar flow was fully developed at the point of insonation. A flow resistor was connected to the outlet of the flow circuit to replicate the resistance of the vascular bed and thereby, damp out any reflections from the tubing connections. In this investigation, a realistic flow-rate waveform was developed based on the spectral analysis of in vivo Doppler ultrasound velocity spectra as seen in Figure 3a. The control signal output by the computer controlled is presented in Figure $3 \mathrm{~b}$. The temperature of the flow phantom and BMF was maintained at $22^{\circ} \mathrm{C} \pm 1^{\circ} \mathrm{C}$ throughout. The error in the velocity produced by the pump was assessed using a volume collection technique and was calculated as $\pm 1.3 \mathrm{~cm} \mathrm{~s}^{-1}$.
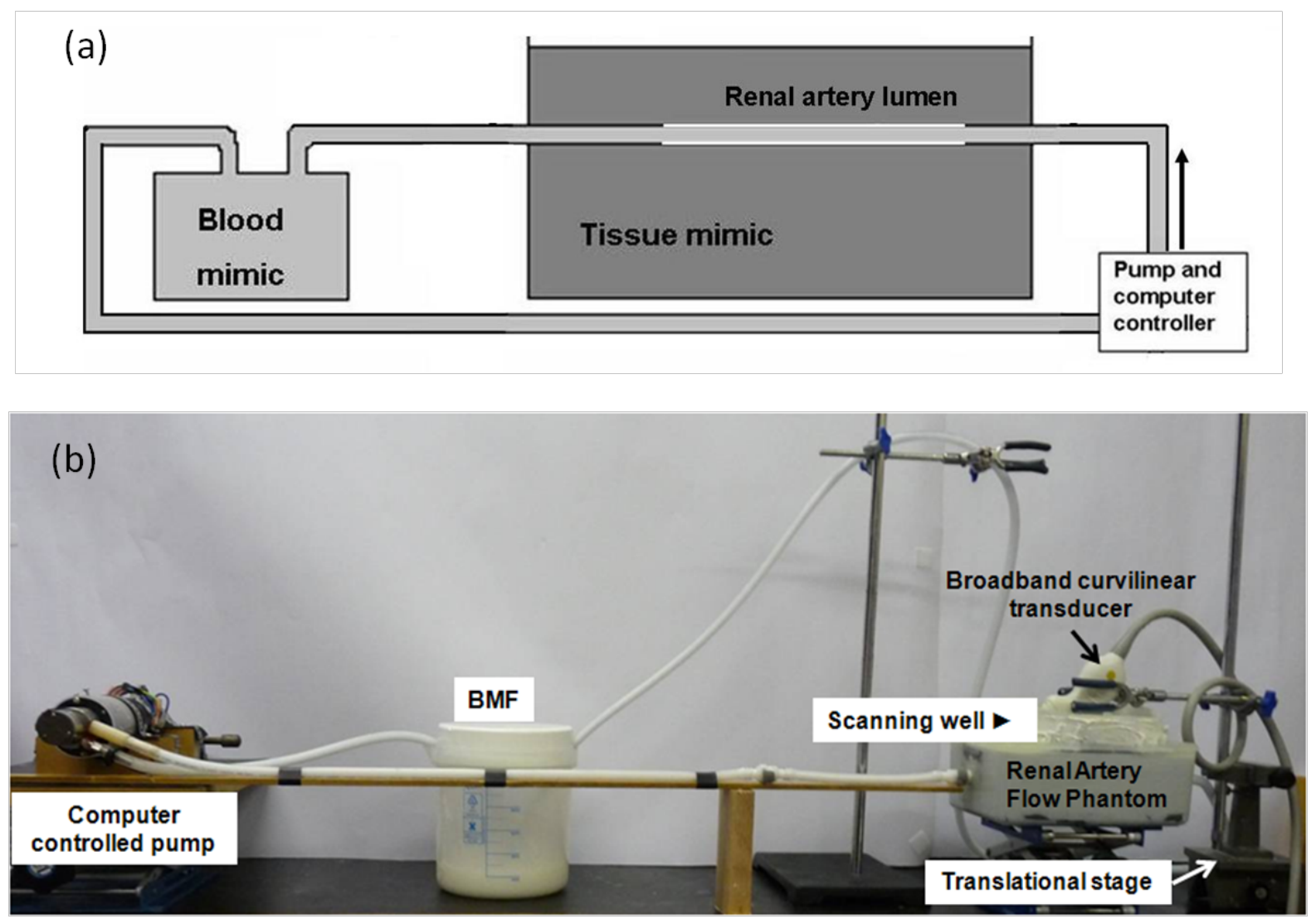

Figure 2: (a) Schematic of flow circuit used for the renal artery flow phantom experiments (b) Renal Artery Flow Phantom and Flow Circuit set-up with the ultrasound transducer secured in the scanning well using a 2-D translation stage. 

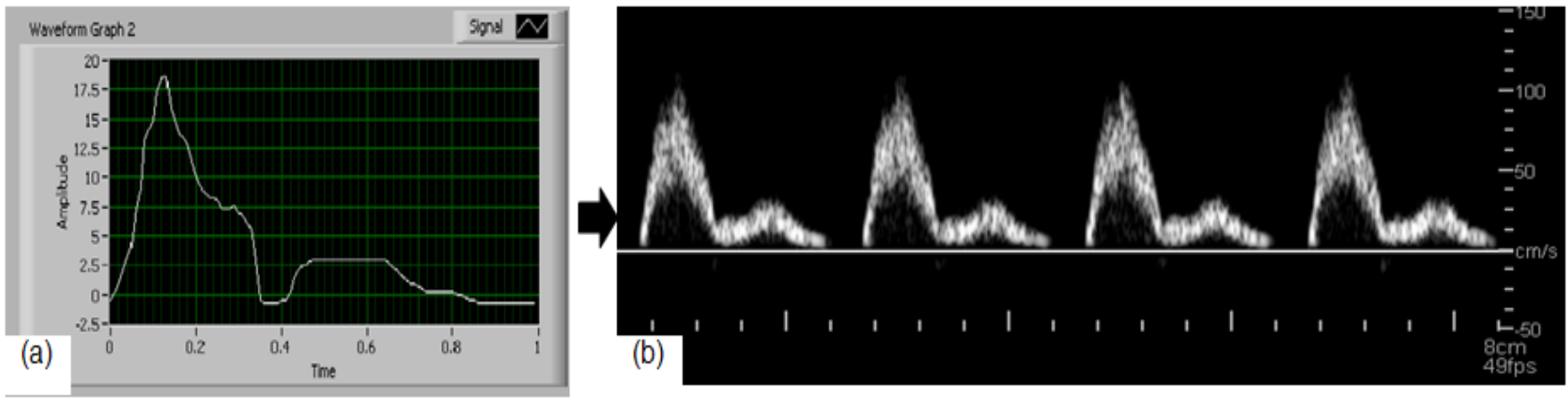

\section{Figure 3: (a) Graphical User Interface (GUI) of the LABVIEW program showing a physiological flow waveform (b) physiological flow waveform response recorded using Pulsed Wave spectral Doppler.}

A Sonoline Antares (Siemens Medical Solutions, Karlsruhe, Germany) ultrasound system with a broadband curvilinear transducer (C5-2), was used for determining the velocity of the BMF using PW Spectral Doppler during the flow experimentations. The curvilinear transducer was clamped to a micrometer manipulator mounted on a $2 \mathrm{D}$ translational stage. A scanning well containing degassed water-glycerol ( $10 \%$ glycerol and $90 \%$ water) solution was used as an acoustic window between the transducer and the renal artery phantom. The transducer was angled to produce a beam-to-vessel angle of $60^{\circ}$ and was positioned such that a $20 \mathrm{~mm}$ layer of the water-glycerol solution separated the transducer face from the phantom surface. The B-mode image was optimised by adjusting the image depth so that the vessel lumen was in the centre of the image and by positioning the focal zone to the depth of the vessel, maximizing the output power (Thermal Index of 1.1 and Mechanical Index (M.I.) of 0.7) while the Time Gain Compensation controls were adjusted to produce a uniform image of the phantom. The Pulsed Wave (PW) Spectral Doppler mode was turned on and the spectral waveform was optimised by adjusting the Doppler Gain to $65 \%$, the wall filter setting to low filter setting $(47 \mathrm{~Hz})$, sample volume size of $1 \mathrm{~mm}$ and the Pulse Repetition Rate was adjusted so that the spectrum encompassed $75 \%$ of the available Spectrum window.

Five peak systolic velocities and five peak diastolic velocities were determined at seven radial positions at $1 \mathrm{~mm}$ intervals across the vessel lumen and averaged, giving a blood velocity profile for a single axial position in the vessel. This procedure was repeated at $1 \mathrm{~mm}$ axial intervals along the vessel length to examine the change in velocity profile with respect to axial position. The radial positions were controlled through Doppler range gate positioning while the axial positions were controlled through the use of a micrometer manipulator adjustment positional system. The physiological velocity profiles were investigated in each stenosis model (concentric $30 \%$ and $50 \%$ and eccentric $25 \%, 35 \%$ and $45 \%$ ) of the of the renal artery flow phantom using this measurement protocol.

A simple method to compute the WSS for steady flow data was presented in Katritsis et al. [33]. This method is based on Poiseuille's law. Starting with Poiseuille's law for volumetric flow rate (Q):

$$
Q=\frac{\pi R^{4}\left(p_{1}-p_{2}\right)}{8 \mu L}
$$

Where $R$ is the lumen radius, $p_{1}$ and $p_{2}$ are the pressure values at the beginning and end of a vessel segment of length $L$, and $\mu$ is the dynamic viscosity. Poiseuille defines WSS in this regime as:

$$
\tau_{w}=\frac{R\left(p_{1}-p_{2}\right)}{2 L}
$$


By combining these equations, an equation expressing WSS in terms of volumetric flow rate is achieved:

$$
\tau_{w}=\frac{4 \mu Q}{\pi R^{3}}
$$

The volumetric flow rate can be taken as the product of the average flow velocity and the crosssectional area of the vessel and, for fully developed and parabolic flow, the average velocity is equal to half the maximum velocity.

This allows the computation of the WSS using the equation [33]:

$$
\tau_{w}=\frac{2 \mu V_{\max }}{R}
$$

Where: $\tau_{w} V_{\max }$ was the peak velocity recorded at the centre of the vessel. This calculation was carried out for each longitudinal position in each vessel to produce longitudinal WSS curves.

\section{Results}

\section{Geometric Accuracy of the Renal Artery Flow Phantoms}

No statistically significant difference was found between the renal artery metal model diameter and the renal lumen diameter for all degrees of concentric and eccentric stenoses, thus confirming that the phantom development method was reproducible. B-mode images of the region of the renal artery flow phantoms displaying the inlet, stenosis region of interest and the bend were acquired using a linear transducer (5-13 MHz) on a Siemens Antares system and are presented in Figure 4(a)(h) for the normal geometry, concentric and eccentric stenosed geometries.

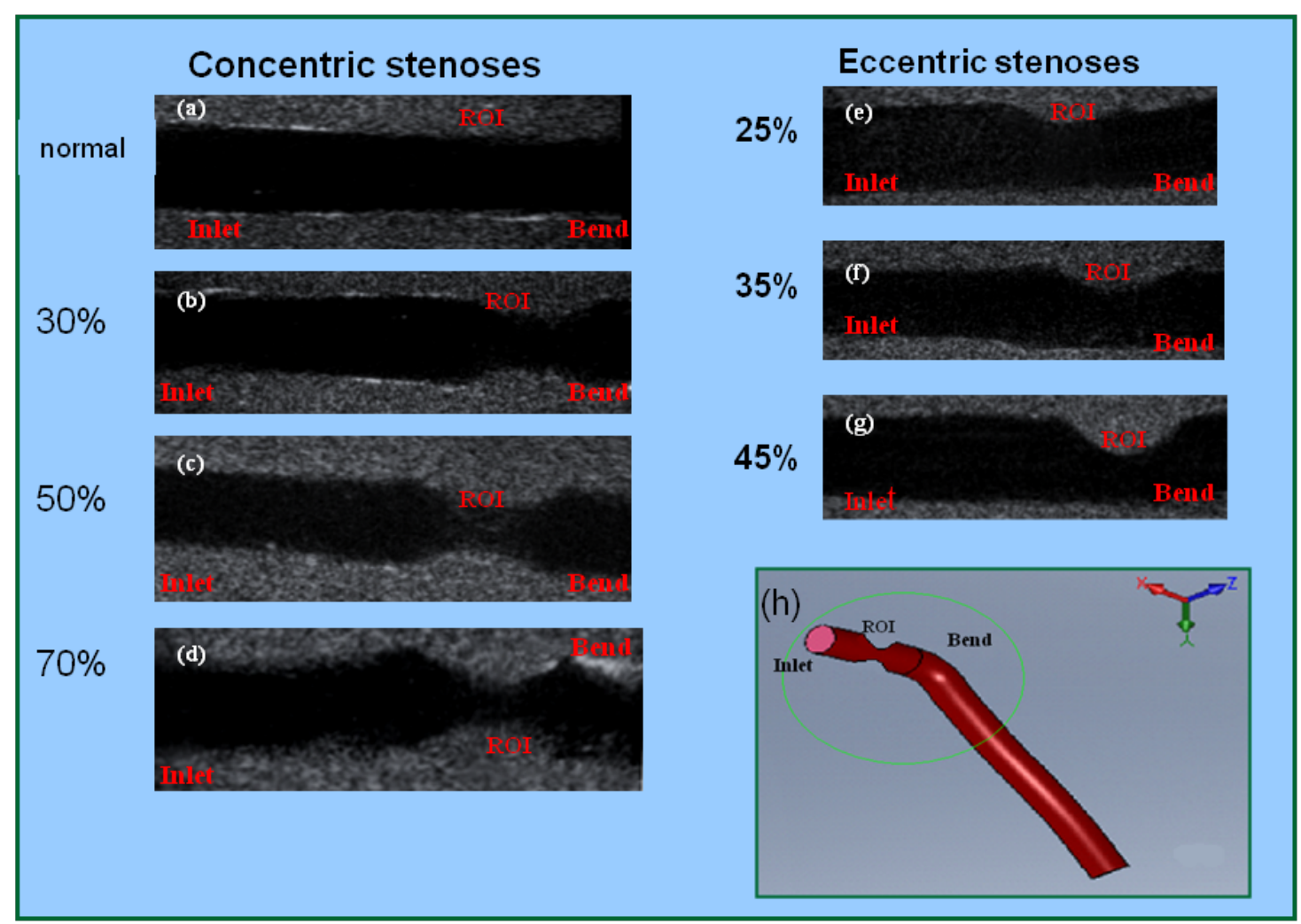

Figure 4: B-mode images of the region of the renal artery flow phantoms displaying the inlet, stenosis region of interest and the bend acquired using linear transducer (5-13 MHz) on a Siemens 


\begin{abstract}
Antares system (a) normal geometry; (b) 30\% concentric stenosis geometry; (c) 50\% concentric stenosis geometry; (d) $70 \%$ concentric stenosis geometry; (e) 25\% eccentric stenosis geometry; (f) 35\% eccentric stenosis geometry; (g) 45\% eccentric stenosis geometry; and (h) 3D geometry of the renal artery lumen indicating the inlet, stenosis region of interest and the bend region.
\end{abstract}

\title{
Geometric Stability Assessment of Stenosis Models
}

The geometrical stability of the range of renal artery flow phantoms was assessed on the basis of visual observations used to identify any signs of TMM fracture or BMF leakage in the flow phantoms. During the assessment, for the initial two hours in the lower grade (normal, concentric 30\% and eccentric $25 \%, 35 \%$ and $45 \%$ ) stenoses flow phantoms, no signs of the TMM rupture or of the BMF leakage were observed and therefore, these renal artery flow phantoms were found to be stable through the entire experimentation procedure. The $50 \%$ wall less renal flow phantom ruptured at the vessel curvature after 3 days of experimentation. As a result, three $50 \%$ wall less renal artery flow phantoms were developed and utilised to complete the flow experiments described in this paper. The renal lumen diameters at the stenosis measured in each renal artery flow phantoms after 1 and 4 hours of continuous physiological flow were observed to be identical and therefore, the stenosis geometry in the renal artery flow phantoms were found to be stable during the flow experimentation. From this assessment of the geometrical stability of the stenosis models, it was evident that the constructed stenosis models of renal artery flow phantoms could be used for the physiological flow experiments using pump velocities between $2-40 \mathrm{~cm} \mathrm{~s}^{-1}$ for all phantom geometries. In principle, the phantoms of lower stenosis degree could be driven at greater pump velocities than highly stenosed phantoms due to the proportionally smaller jet velocities.

\section{Ultrasound Flow Data}

For each phantom, a velocity profile contour plot was generated using the peak systolic velocities recorded at $1 \mathrm{~mm}$ intervals radially across the vessel. These measurements were repeated axially along the vessel length so as to encompass the regions of normal flow, vessel curvature, and vessel stenosis.

\section{Symmetrical stenosis models}

In Figure 5, the typical flow profile in a vessel exhibiting no arterial stenosis is presented while in Figure 6 the velocity profile in vessels which have 3D symmetric stenoses of $30 \%$ and $50 \%$ are presented. To facilitate comparison of the velocity profiles between phantoms, these plots were produced with the same velocity scale $\left(35-90 \mathrm{~cm} \mathrm{~s}^{-1}\right)$.

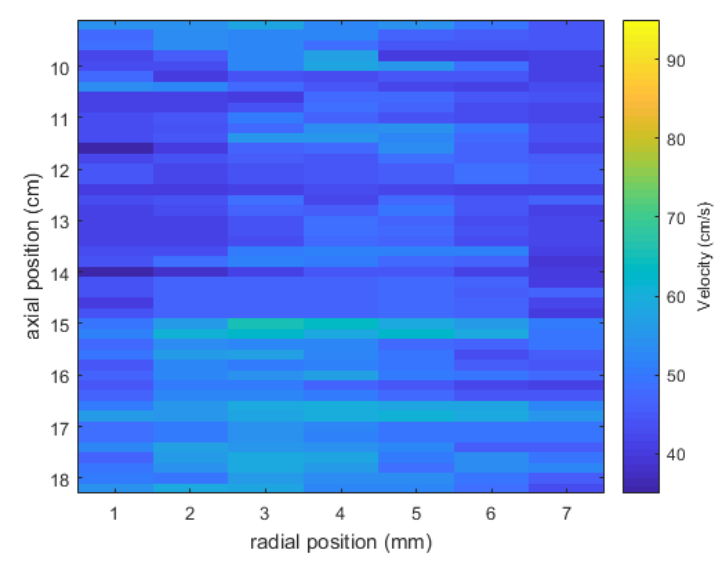

Figure 5: Physiological flow velocity profiles in the $0 \%$ stenosis renal artery phantom 

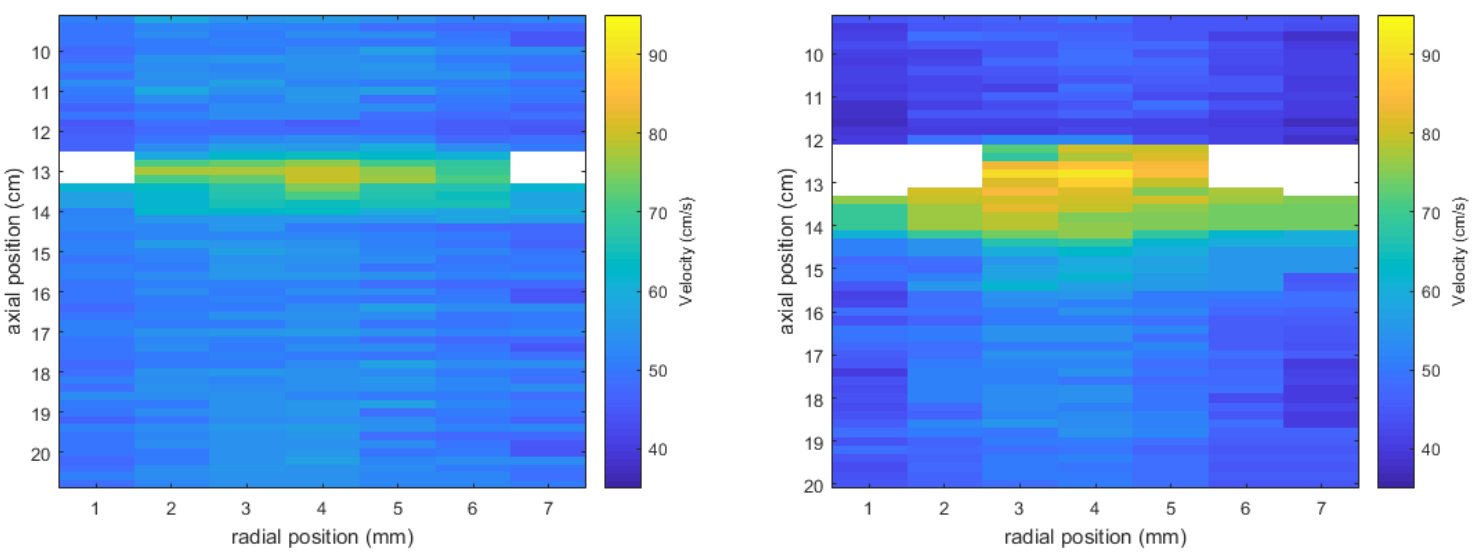

Figure 6: Physiological flow velocity profiles in the $30 \%$ and $50 \%$ stenosis renal artery phantoms

The physiological flow profiles at the stenosis and curvature regions of the vessel were of most interest and therefore, were examined in closer detail with higher velocity resolution. The velocity profile behaviour in the $0 \%$ or normal phantom at both the stenosis and curvature regions is presented in Figure 7. Figures 8 and 9 presents the flow profiles in the stenosis and curvature regions of the $30 \%$ and $50 \%$ flow phantoms, respectively, the axial extent of the curvature is indicated with a red box.
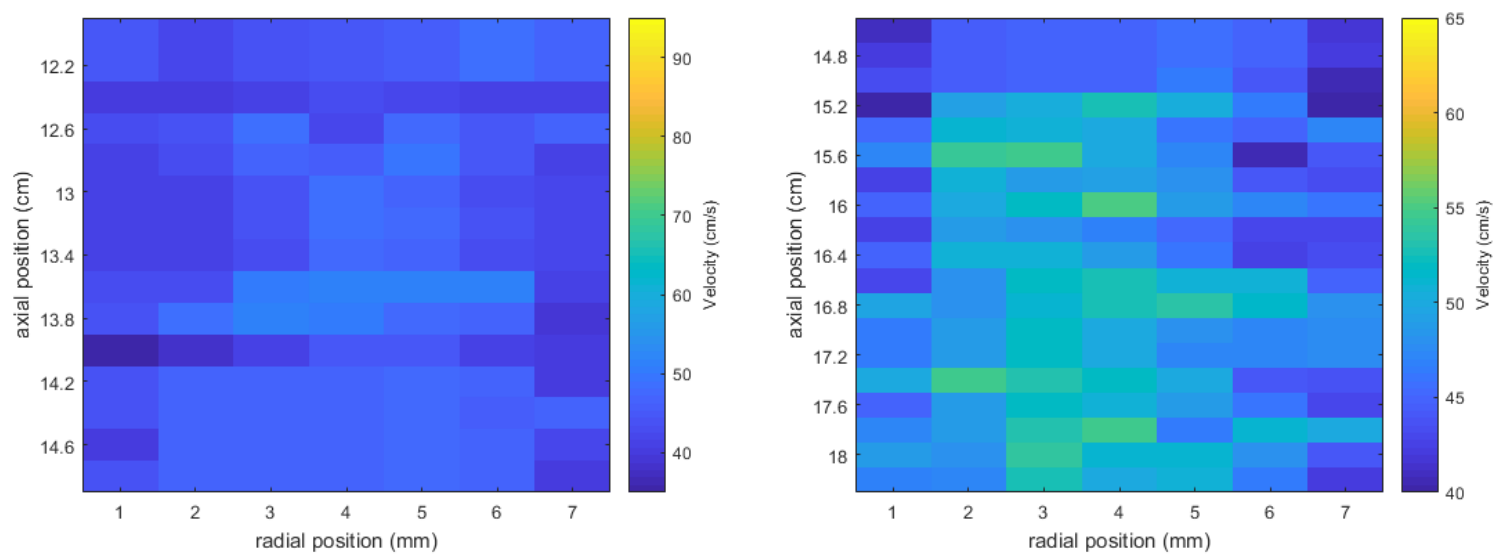

Figure 7: Physiological flow profiles for the $0 \%$ control renal artery phantom at the stenosis and curvature regions respectively
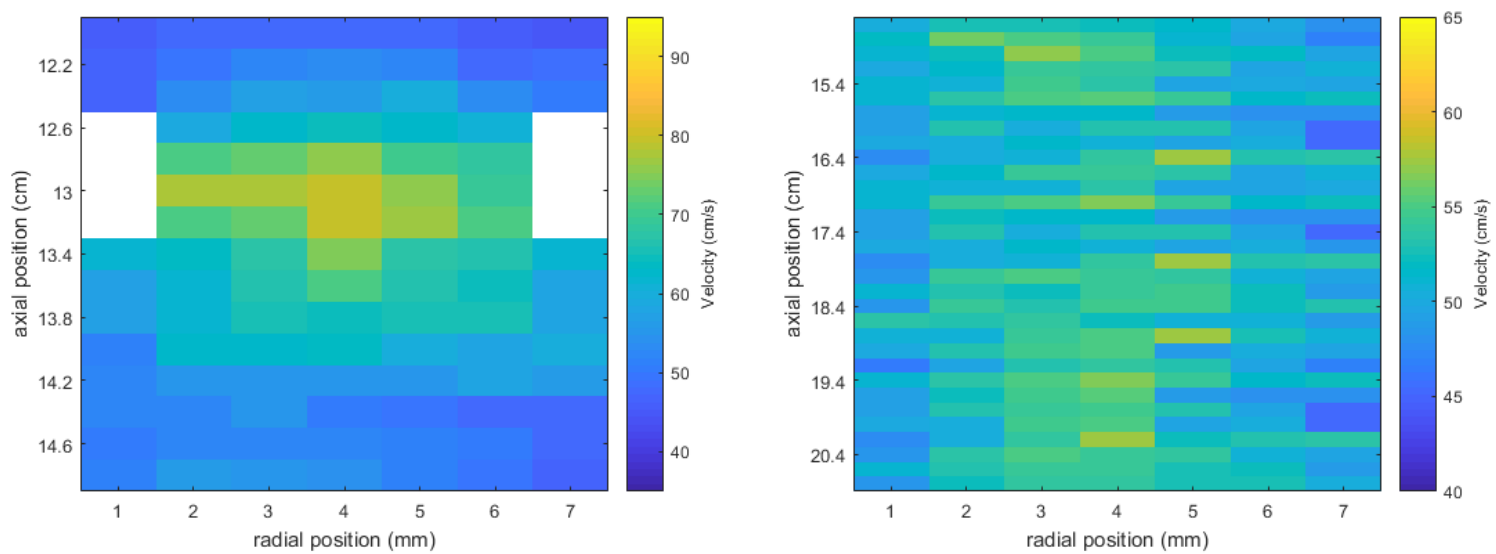
Figure 8: Physiological flow profiles for the $30 \%$ symmetric renal artery phantom at the stenosis and curvature regions respectively
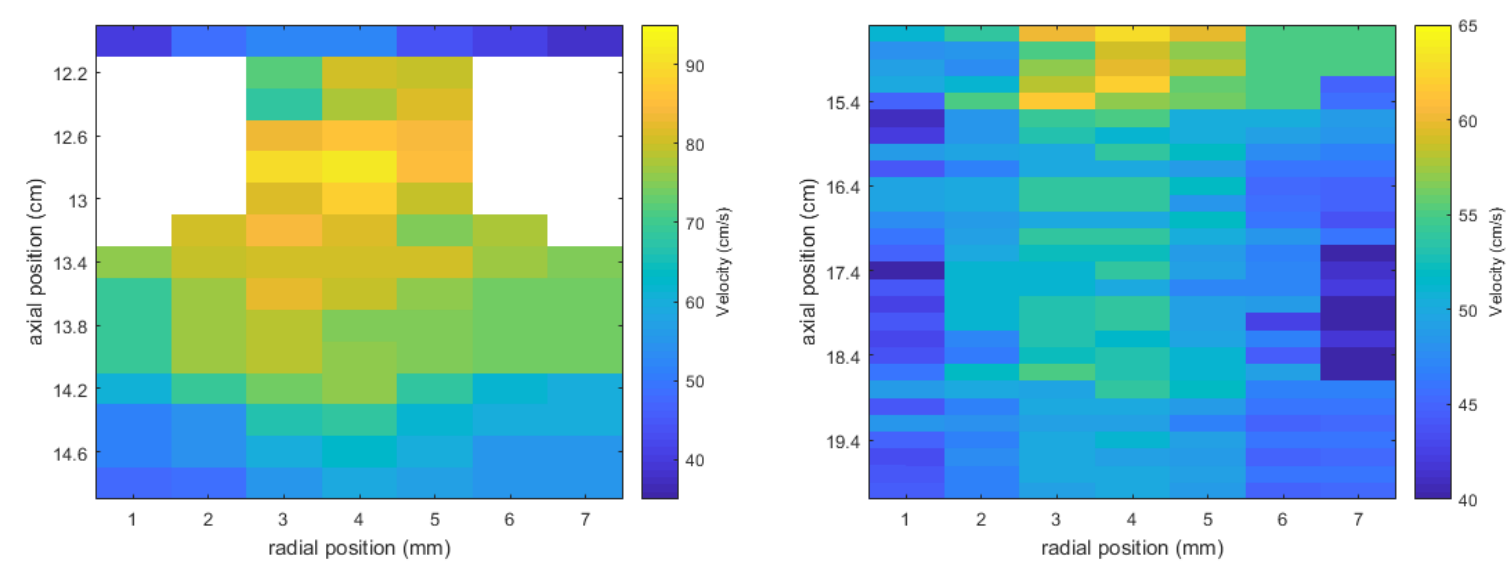

Figure 9: Physiological flow profiles for the $50 \%$ symmetric renal artery phantom at the stenosis and curvature regions respectively

The velocities measured in the normal $(0 \%)$ renal artery flow phantom were in the region of $35-60$ $\mathrm{cm} \mathrm{s}^{-1}$ for the full vessel length. The curvature region in this phantom produced an increase in velocities in the range of $40-60 \mathrm{~cm} \mathrm{~s}^{-1}\left(5-10 \mathrm{~cm} \mathrm{~s}^{-1}\right.$ higher) and in addition the velocities recorded in this region showed a small degree of skewing toward the outer wall of the vessel, i.e. the section of the vessel with the largest radius of curvature. The velocities measured in the $30 \%$ renal artery flow phantom were in the range of $45-80 \mathrm{~cm} \mathrm{~s}^{-1}$ for the entire vessel, with the majority of the higher velocities were measured in the stenosis region $\left(55-80 \mathrm{~cm} \mathrm{~s}^{-1}\right)$ with some slower flow in the curvature region $\left(45-60 \mathrm{~cm} \mathrm{~s}^{-1}\right)$. Again, this region exhibited a slight skewing towards the outer vessel wall. The velocities in the $50 \%$ renal artery flow phantom were in the range of $40-90 \mathrm{~cm} \mathrm{~s}^{-1}$ and, similarly to the $30 \%$ renal artery flow phantom, the majority of the high velocities were measured in the stenosis region $\left(60-90 \mathrm{~cm} \mathrm{~s}^{-1}\right)$ with mainly lower velocities found in the curvature region $\left(40-55 \mathrm{~cm} \mathrm{~s}^{-1}\right)$ and some slight skewing towards the outer vessel wall.

In all phantoms the measured velocities were observed to increase in all radial planes up until the end of the vessel curvature. After the curvature region, the observed shift of high velocities to the outer wall was seen to correct itself, with the higher velocities returning to the central axis.

Indicating with stenosis $<50 \%$ the haemodynamical changes are local to the stenosed region.

\section{Asymmetric stenosis models}

Figure 11 shows the physiological velocity profiles in the $25 \%, 35 \%$, and $45 \%$ asymmetrical renal artery flow phantoms. 

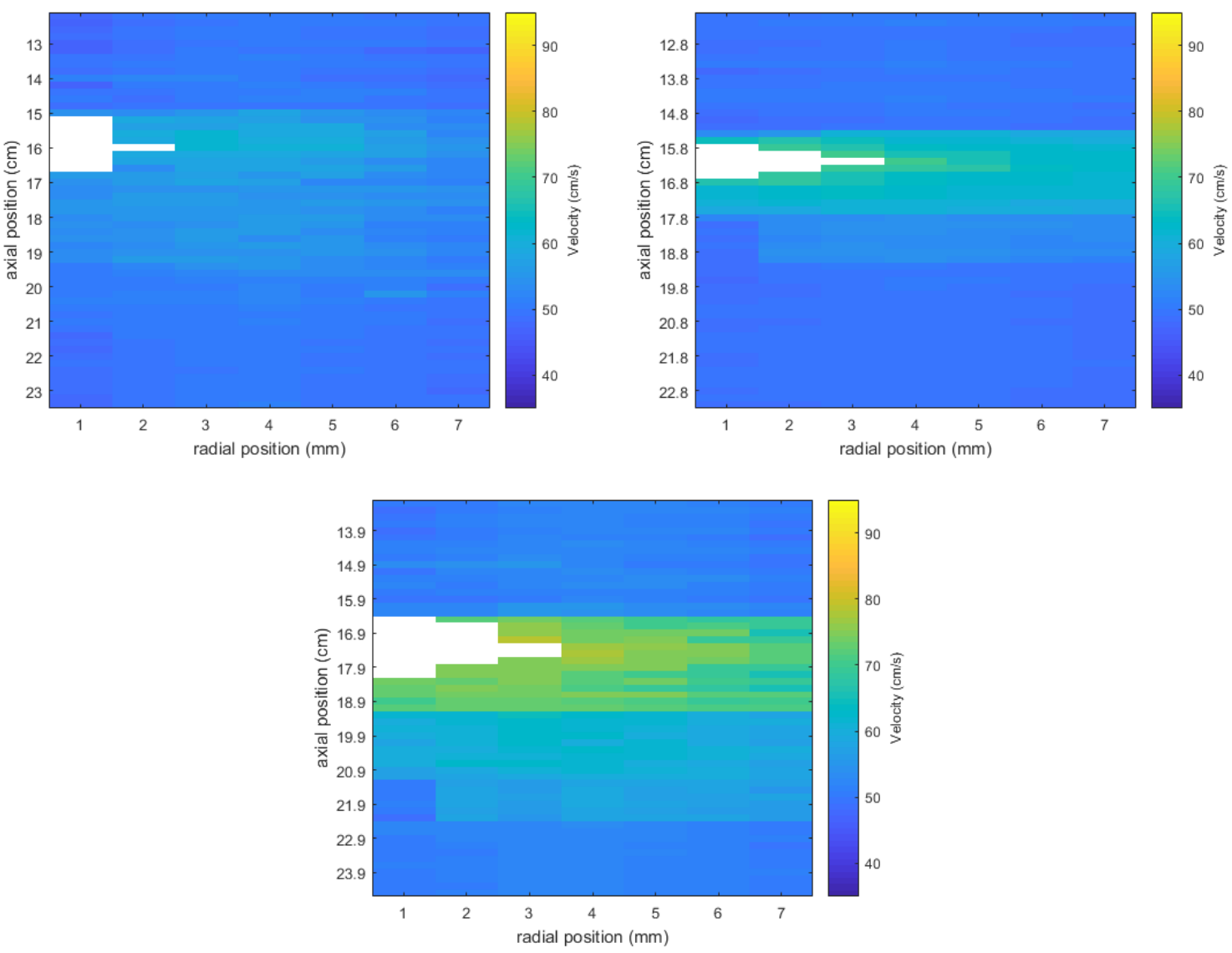

Figure 10: Physiological flow velocity profiles in the $25 \%, 35 \%$, and $45 \%$ stenosis renal artery phantoms

As with the symmetrical renal artery phantoms, the stenosis and curvature regions of the asymmetrical renal artery phantoms were of most interest and so they were examined in closer detail with higher velocity resolution. The velocity profile behaviour for the stenosis and curvature regions of the $25 \%, 35 \%$, and $45 \%$ asymmetric phantoms are shown in Figures $12-14$, respectively.
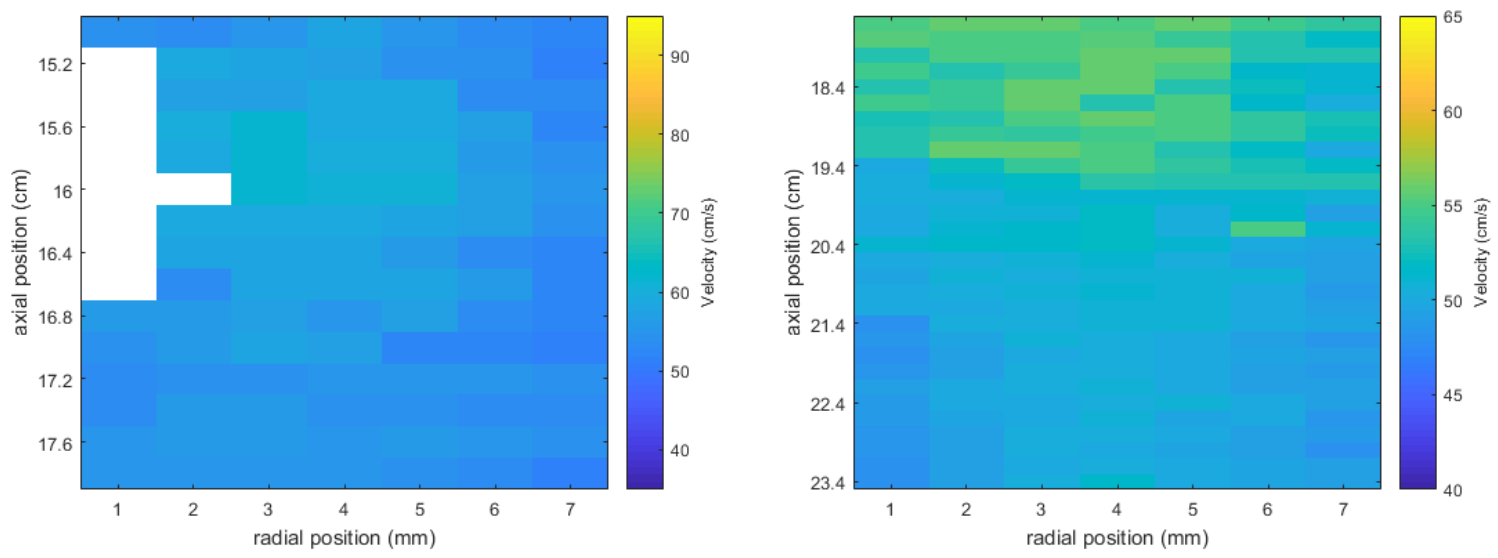

Figure 11: Physiological flow profiles for the $25 \%$ asymmetric renal artery phantom at the stenosis and curvature regions respectively 

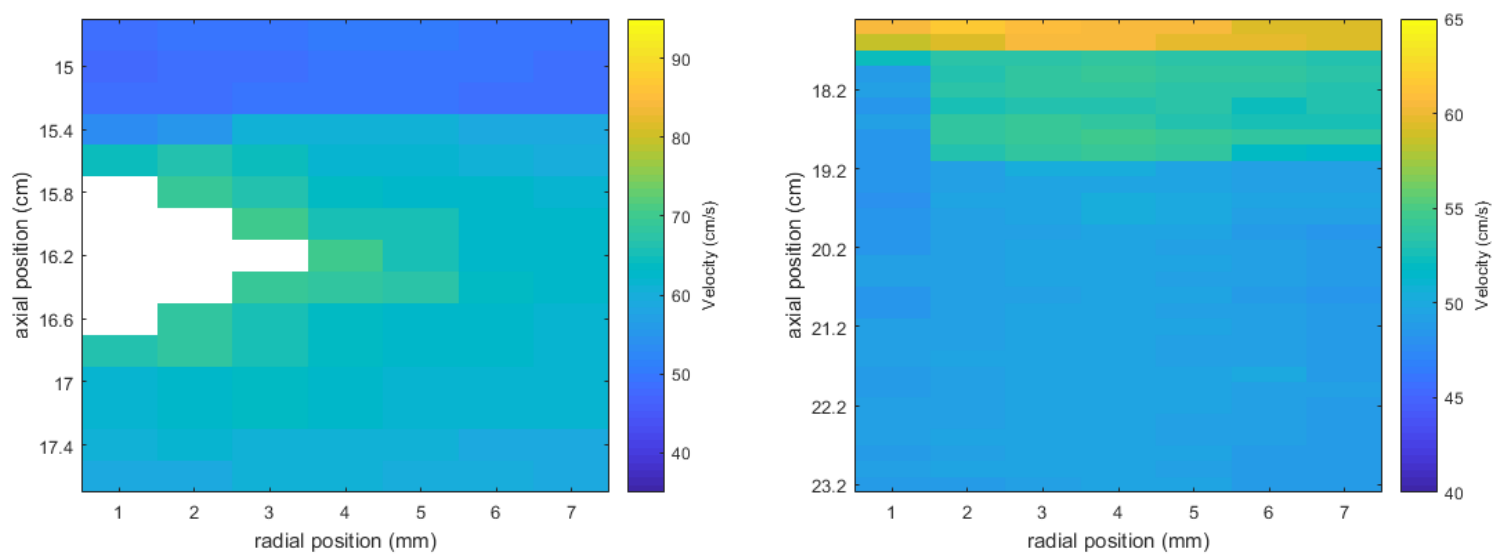

Figure 12: Physiological flow profiles for the 35\% asymmetric renal artery phantom at the stenosis and curvature regions respectively
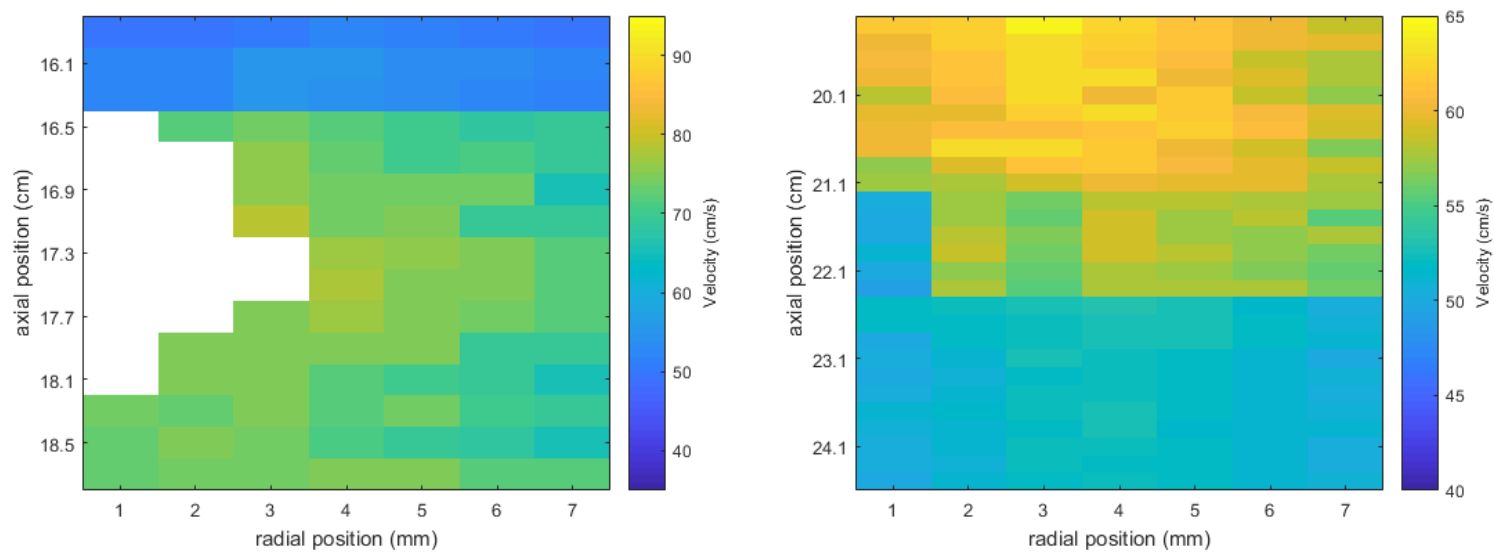

Figure 13: Physiological flow profiles for the 45\% asymmetric renal artery phantom at the stenosis and curvature regions respectively

For the $25 \%$ asymmetrical stenosis phantom, the typical flow rates were in the range of $45-62 \mathrm{~cm} \mathrm{~s}$ ${ }^{1}$. For the $35 \%$ asymmetrical stenosis phantom the typical velocity ranges were slightly higher with a range of $48-70 \mathrm{~cm} \mathrm{~s}^{-1}$. As expected, the highest velocity range was found in the $45 \%$ asymmetrical stenosis phantom, with typical velocities in the range $50-81 \mathrm{~cm} \mathrm{~s}^{-1}$.

As with the symmetrical renal artery phantoms, the highest velocities in each phantom were localised in the stenosis regions. For the $25 \%$ asymmetrical phantom the stenosis region exhibited velocities in the range of $54-62 \mathrm{~cm} \mathrm{~s}^{-1}$ compared to the curvature region range of $50-55 \mathrm{~cm} \mathrm{~s}^{-1}$. The $35 \%$ asymmetrical phantom exhibited velocities in the range of $57-72 \mathrm{~cm} \mathrm{~s}^{-1}$ for the stenosis region compared to a range of $48-50 \mathrm{~cm} \mathrm{~s}^{-1}$ in the vessel curvature. Finally, the $45 \%$ asymmetrical phantom featured typical velocities in the range of $60-81 \mathrm{~cm} \mathrm{~s}^{-1}$ for the stenosis region compared to $50-53 \mathrm{~cm} \mathrm{~s}^{-1}$ for the vessel curvature.

Similarly to the symmetrical renal artery phantoms, the asymmetrical phantoms were observed to have a skewing of high velocities towards the outer vessel wall, although the magnitude of the skewing was more pronounced for the asymmetrical stenosis renal artery phantoms and the velocity profile behaviour recovered more slowly, over a longer axial distance. 
The velocity data of all the vessels was analysed to produce WSS curves as described previously. These curves are displayed graphically in Figure 14. The curves for the symmetrical stenoses had a sharp increase in WSS values at the stenosis region and remained approximately at this peak level for $1-2 \mathrm{~cm}$ before exhibiting a rapid decrease in WSS.

Conversely, the asymmetric stenoses displayed a brief peak of relatively high WSS values that were both spatially shorter and exhibiting a smaller magnitude compared to the symmetric stenoses. However, after the peak of WSS values, the overall WSS slowly recovered back to normal levels on a longer spatial distance than the symmetrical stenoses.
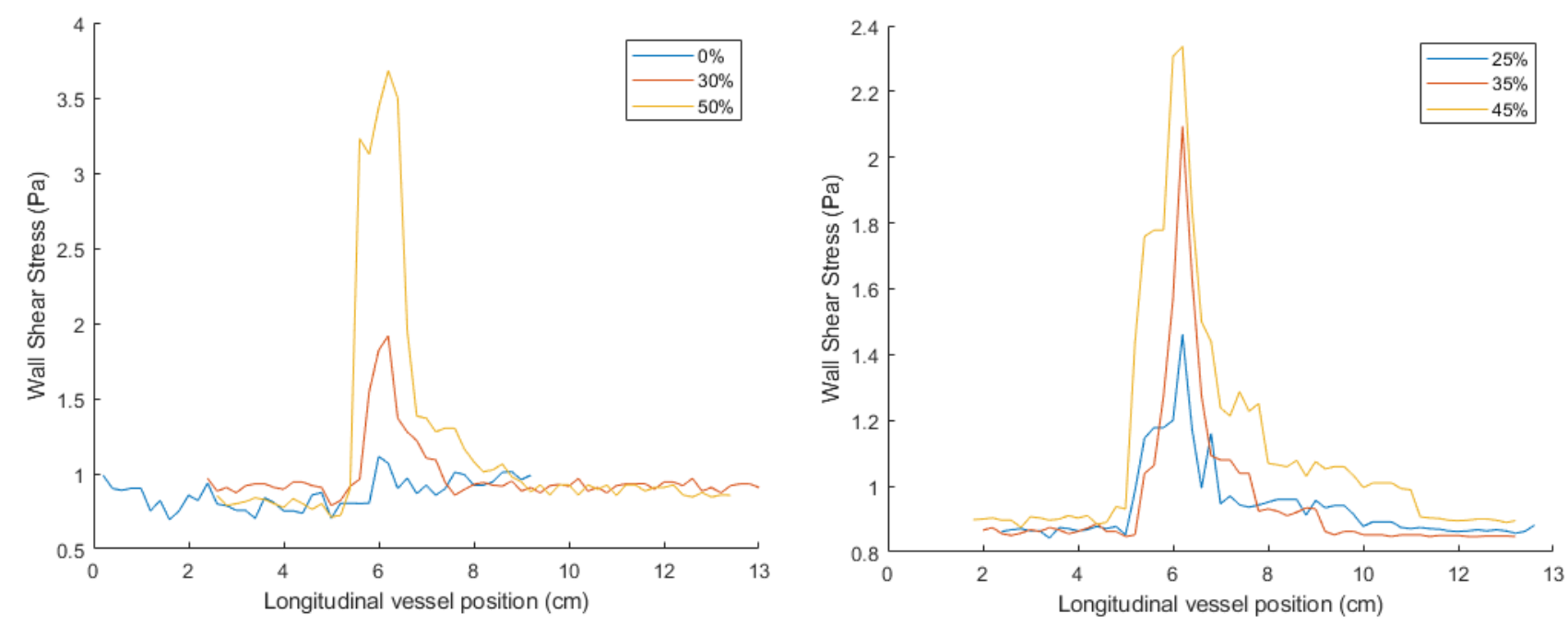

Figure 14: Wall shear stress curves generated for symmetric (left) and asymmetric (right) stenosed vessels. The absolute magnitude of WSS increase for symmetric stenoses was larger than asymmetric at approximately equivalent scales. Additionally, the WSS values of the symmetric stenosed vessels maintained a peak value for a greater distance than the asymmetric stenosed vessels and fell off rapidly while the asymmetric stenosed vessels were observed to peak rapidly and slowly return to normal values.

\section{Discussion}

RAS is a serious condition associated with a number of life threatening and life limiting conditions. Furthermore, the treatment of RAS for current diagnostic practice is largely surgical and inherently complex [34] due to diagnosing advanced disease. Therefore, the early diagnosis of RAS is of paramount importance to allow for the exploration of less invasive treatment options.

The criteria for selecting the most suitable technique for diagnosing patients with suspicion of RAS are currently ill-defined, in terms of the required spatial resolution and velocity detection performance for quantification of the vascular geometry and characterisation of the blood flow. Doppler ultrasound represents a potential avenue for the bridging of the diagnostic gap, particularly for the quantification of blood velocity profiles with a vessel at high risk locations throughout the body. Using this information, the clinical biomarker, wall shear stress, could be calculated; giving an important insight into overall endothelial health. This study represents a proof of concept regarding whether current Doppler ultrasound has sufficient velocity and spatial resolution for full quantifying velocity profiles across a vessel and, therefore, could potentially be used for deriving wall shear stress. Doppler ultrasound is uniquely placed for further advancement in this area with modern technological advancements such as UltraFast imaging on the Aixplorer ultrasound scanner 
(supersonic Imagine, France) which allows for B-mode image acquisitions up to 1000 frames per second, in addition to full Doppler velocity quantification of the entire imaging region with a factor of 16 reduction in acquisition time [35], [36].

As such, Doppler ultrasound was used to determine velocity profiles and to investigate whether local changes in haemodynamic profiles could be detected in the presence of a $<50 \%$ stenosis. This was achieved through the use of a range of anatomically realistic renal artery (normal, 30\%, $50 \%$ symmetrical and $25 \%, 35 \%$, and $45 \%$ asymmetric) flow phantom. Previous studies have largely focused on the measurement of the peak systolic velocity as a measurement of stenosis degree [37], [38]. The results of this work were similar to complete flow mapping studies of other modalities such as particle image velocimetry (PIV). An example of such a work was carried out by Kefayati \& Poepping [39]. In this work, carotid artery bifurcation models were assembled for use in PIV flow studies. The models featured "concentric" symmetrical stenoses of $50 \%$ and $70 \%$ as well as a control healthy model. Despite the models exhibiting larger stenosis degrees than this work, the results are similar with a reported complex helical flow pattern immediately distal to the stenosis followed by a relaminarisation of flow downstream with the turbulence covering a longer distance for the higher stenosis degree. Another option for the whole vessel evaluation of flow is the use of phase contrast MRI. Kadbi et al. used phase contrast MRI to quantify the blood velocity profile for a $90 \%$ symmetrical stenosed vascular phantom [40]. In this study, Kadbi et al. detected similar results to that of this work and that of Kefayati \& Poepping in terms of turbulence distal to the stenosis region followed by relaminarisation downstream. Kadbi et al. did not detect an asymmetric skewing of blood velocity due to the fact that the phantom used in their study did not exhibit a curve nor asymmetrical stenoses.

It was evident that the shape and the symmetry of the stenoses of lower grades $(\leq 45 \%)$ affected the flow velocity profiles and that Doppler ultrasound was capable of detecting this change. The velocity profiles presented several key characteristics which would prove useful in the diagnosis of early stage RSA (<50\%). Firstly, the skewing of the velocity profile towards the outer vessel wall in the curvature regions of the vessel (particularly visible in Figure 12) was more pronounced in the presence of an asymmetrical stenosis and the haemodynamic changes were observed at a greater axial distance from the stenosed region. This was similar to the results in a patient specific flow simulation by Lee et al. where there was a strong asymmetric skew of blood velocity in the presence of a $45 \%$ asymmetric carotid stenosis [41]. Additionally, the magnitude of velocity elevation was observed to be an indicator for the degree of stenosis. Previously peak systolic velocity has been used as an indicator of arterial stenosis, however it has typically only been used to diagnose the presence of stenoses of relatively large degree (>50\%) [5]. Finally, the WSS curves generated from this data showed a clear significant peak for every stenosis type and could even potentially be used to differentiate between symmetric and asymmetric stenoses. These results indicated that the use of Doppler ultrasound can provide insight into the degree and morphology of renal artery stenoses for relatively low stenosis degrees.

An example of a comparable analysis carried out using ultrasound can be found in Yiu et al. [42]. Yiu et al. presents a new technique in the assessment of velocity instability, Doppler ultrasound bandwidth imaging (DUBI). DUBI allows for the mapping of areas of flow disturbance by measuring bulk increases in Doppler spectral broadening which indicates a range of velocities present in a voxel and, therefore, a region of flow instability. This technique was used on a series of carotid bifurcation models featuring $50 \%$ and $75 \%$ asymmetric stenoses as well as a $0 \%$ control model. These flow experiments again showed comparable results to those found in this study, with regions of instability 
immediately distal to the stenoses and a strong skew of higher velocities to the outer wall following the stenoses.

Although the data presented from each of these renal artery phantoms provided diagnostically useful data regarding the haemodynamic behaviour near $<50 \%$ stenoses which would be of great benefit in diagnosis of a stenosis at an earlier stage in development, it would not be possible to use the methodology in a clinical setting. This is due to the limitations of the ultrasound system used which required that that velocity profiles were measured over a period of hours through the systematic translation of the transducer through the radial and axial positions within the vessel and were also averaged across different peaks in the simulated cardiac cycle. However, with the introduction of more advanced Doppler ultrasound techniques such as UltraFast Doppler [36] there is potential for the data to be collected over this entire axial region within a cardiac cycle and for the Doppler gate to be translated radially to acquire a sufficient number of samples to produce such velocity profiles within a few seconds. This would provide a more detailed assessment of the vessel of interest and potentially provide a means of earlier detection of low grade stenoses $(<50 \%)$. The investigation of the use of UltraFast Doppler in this type of assessment warrants investigation particularly for the assessment of wall shear stress.

A limitation of this study was the use of wall-less flow phantoms, however, the relative change in the velocity profiles over the phantom geometry was the major interest point of this study and this was achieved using the wall-less flow phantoms. We plan to investigate the incorporation of walled vessels in similar types of flow phantoms in the future once a suitable vessel mimicking material has been developed which appropriately mimics the acoustic and mechanical properties of the arterial tissue.

In order to ensure that the optimum resolution in the lateral and slice directions was achieved every effort was taken such that the Doppler measurements were acquired as close to the transducer focus as possible. However, an additional limitation of this study was that the sample size for each measurement may have deviated from the displayed resolution which would result in additional velocity data being introduced into these sample sizes, in particular where measurements were close to the vessel wall. The purpose of this work was to evaluate whether detailed velocity profiles could be measured and, therefore, used to detect low grade stenosis. We have demonstrated this, albeit with an inherent limitation in the ultrasound system used in this work. Indeed, novel advanced imaging techniques, such as UltraFast technology available on the Aixplorer, now reportedly demonstrate high frame rates with axial and lateral resolutions as low as $0.1 \mathrm{~mm}$ [36].Thus, using such technology and the technique proposed in this work, the early diagnosis of renal artery stenosis is a real possibility.

\section{Conclusion}

The use of Doppler ultrasound for the investigation of the physiological velocity profiles in anatomically realistic renal artery flow phantoms with a range of symmetrical and asymmetrical stenoses was investigated. The developed renal artery flow phantoms closely mimicked the in-vivo situation. Through the use of these flow systems it was possible to experimentally determine changes in the systolic velocity measurements and physiological velocity profiles in the presence of stenosis of different types and degrees as well as an area of curvature. The magnitude of change in the systolic velocity measurements and velocity profile characteristics in presence of the lower degree stenosis indicated that the systolic velocity profiles can be effectively used to detect the 
stenoses of lower grades. This type of assessment could provide an early diagnosis of renal artery stenosis thereby, presenting the opportunity for drug treatments to be used in the treatment which are less invasive and provide a better clinical outcome. 


\section{References}

[1] R. D. Safian and S. C. Textor, "Renal-Artery Stenosis," N. Engl. J. Med., vol. 344, no. 6, pp. 431-442, Feb. 2001.

[2] J. Radermacher and R. Brunkhorst, "Diagnosis and treatment of renovascular stenosis -a cost - benefit analysis," Nephrol Dial Transpl., vol. 13, pp. 2761-2767, 1998.

[3] H. R. Reynolds et al., "Frequency of severe renal artery stenosis in patients with severe thoracic aortic plaque.," Am. J. Cardiol., vol. 94, no. 6, pp. 844-6, Sep. 2004.

[4] C. Rountas et al., "Imaging Modalities for Renal Artery Stenosis in Suspected Renovascular Hypertension: Prospective Intraindividual Comparison of Color Doppler US, CT Angiography, GD-Enhanced MR Angiography, and Digital Substraction Angiography," Ren. Fail., vol. 29, no. 3, pp. 295-302, Jan. 2007.

[5] V. Aboyans et al., "2017 ESC Guidelines on the Diagnosis and Treatment of Peripheral Arterial Diseases, in collaboration with the European Society for Vascular Surgery (ESVS)," Eur. J. Vasc. Endovasc. Surg., Aug. 2017.

[6] C. G. Caro, J. M. FitzGerald, and R. C. Schroter, "Atheroma and arterial wall shear Observation, correlation and proposal of a shear dependent mass transfer mechanism for atherogenesis," Proc. R. Soc. London. Ser. B. Biol. Sci., vol. 177, no. 1046, pp. 109-133, Feb. 1971.

[7] B. K. Bharadvaj, R. F. Mabon, and D. P. Giddens, "Steady flow in a model of the human carotid bifurcation. Part I-Flow visualization," J. Biomech., vol. 15, no. 5, pp. 349-362, Jan. 1982.

[8] C. K. Zarins, D. P. Giddens, B. K. Bharadvaj, V. S. Sottiurai, R. F. Mabon, and S. Glagov, "Carotid bifurcation atherosclerosis. Quantitative correlation of plaque localization with flow velocity profiles and wall shear stress.," Circ. Res., vol. 53, no. 4, pp. 502-514, Oct. 1983.

[9] M. Motomiya and T. Karino, "Flow patterns in the human carotid artery bifurcation.," Stroke, vol. 15, no. 1, pp. 50-56, Jan. 1984.

[10] T. Asakura and T. Karino, "Flow patterns and spatial distribution of atherosclerotic lesions in human coronary arteries.," Circ. Res., vol. 66, no. 4, pp. 1045-1066, Apr. 1990.

[11] A. M. Malek, S. L. Alper, and S. Izumo, "Hemodynamic Shear Stress and Its Role in Atherosclerosis," JAMA, vol. 282, no. 21, p. 2035, Dec. 1999.

[12] H. Eklöf et al., "A prospective comparison of duplex ultrasonography, captopril renography, MRA, and CTA in assessing renal artery stenosis," Acta radiol., vol. 47, no. 8, pp. 764-774, Oct. 2006.

[13] G. M. Soares, T. P. Murphy, M. S. Singha, A. Parada, and M. Jaff, "Renal Artery Duplex Ultrasonography as a Screening and Surveillance Tool to Detect Renal Artery Stenosis," J. Ultrasound Med., vol. 25, no. 3, pp. 293-298, Mar. 2006.

[14] G. J. Williams et al., "Comparative Accuracy of Renal Duplex Sonographic Parameters in the Diagnosis of Renal Artery Stenosis: Paired and Unpaired Analysis," Am. J. Roentgenol., vol. 188, no. 3, pp. 798-811, Mar. 2007.

[15] J. E. Browne, A. J. Watson, P. R. Hoskins, and A. T. Elliott, "Validation of a sensitivity performance index test protocol and evaluation of colour Doppler sensitivity for a range of ultrasound scanners.," Ultrasound Med. Biol., vol. 30, no. 11, pp. 1475-83, Nov. 2004.

[16] D. M. King, C. M. Moran, and J. E. Browne, "Comparative review of imaging methods used for 
diagnosing renal artery stenosis," Ultrasound, vol. 20, no. 3, pp. 135-141, Aug. 2012.

[17] R. Frayne et al., "A geometrically accurate vascular phantom for comparative studies of x-ray, ultrasound, and magnetic resonance vascular imaging: construction and geometrical verification," Med. Phys., vol. 20, no. 2, pp. 415-425, Mar. 1993.

[18] W. Dabrowski, J. Dunmore-Buyze, R. N. Rankin, D. W. Holdsworth, and A. Fenster, "A real vessel phantom for imaging experimentation," Med. Phys., vol. 24, no. 5, pp. 687-693, May 1997.

[19] L. Allard, G. Soulez, B. Chayer, Z. Qin, D. Roy, and G. Cloutier, "A multimodality vascular imaging phantom of an abdominal aortic aneurysm with a visible thrombus," Med. Phys., vol. 40, no. 6Part1, p. 063701, May 2013.

[20] D. M. King, M. Ring, C. M. Moran, and J. E. Browne, "Development of a range of anatomically realistic renal artery flow phantoms.," Ultrasound Med. Biol., vol. 36, no. 7, pp. 1135-44, Jul. 2010.

[21] D. M. King, A. J. Fagan, C. M. Moran, and J. E. Browne, "Comparative imaging study in ultrasound, $\mathrm{MRI}, \mathrm{CT}$, and DSA using a multimodality renal artery phantom; Comparative imaging study in ultrasound, MRI, CT, and DSA using a multimodality renal artery phantom," 2011.

[22] K. V Ramnarine, T. Anderson, and P. R. Hoskins, "Construction and geometric stability of physiological flow rate wall-less stenosis phantoms.," Ultrasound Med. Biol., vol. 27, no. 2, pp. 245-50, Feb. 2001.

[23] J. Bale-Glickman, K. Selby, D. Saloner, and O. Savaş, "Experimental Flow Studies in ExactReplica Phantoms of Atherosclerotic Carotid Bifurcations Under Steady Input Conditions," J. Biomech. Eng., vol. 125, no. 1, p. 38, Feb. 2003.

[24] S. Meagher, T. L. Poepping, K. V Ramnarine, R. A. Black, and P. R. Hoskins, "Anatomical flow phantoms of the nonplanar carotid bifurcation, part II: experimental validation with Doppler ultrasound.," Ultrasound Med. Biol., vol. 33, no. 2, pp. 303-10, Feb. 2007.

[25] T. L. Poepping, H. N. Nikolov, M. L. Thorne, and D. W. Holdsworth, "A thin-walled carotid vessel phantom for Doppler ultrasound flow studies.," Ultrasound Med. Biol., vol. 30, no. 8, pp. 1067-78, Aug. 2004.

[26] T. L. Poepping, H. N. Nikolov, R. N. Rankin, M. Lee, and D. W. Holdsworth, "An in vitro system for Doppler ultrasound flow studies in the stenosed carotid artery bifurcation.," Ultrasound Med. Biol., vol. 28, no. 4, pp. 495-506, Apr. 2002.

[27] S. K. Aytac, H. Yigit, T. Sancak, and H. Ozcan, "Correlation Between the Diameter of the Main Renal Artery and the Presence of an Accessory Renal Artery Sonographic and Angiographic Evaluation," 2002.

[28] C. J. P. M. Teirlinck et al., "Development of an example flow test object and comparison of five of these test objects, constructed in various laboratories," Ultrasonics, vol. 36, no. 1-5, pp. 653-660, Feb. 1998.

[29] J. E. Browne, K. V. Ramnarine, A. J. Watson, and P. R. Hoskins, "Assessment of the acoustic properties of common tissue-mimicking test phantoms," Ultrasound Med. Biol., vol. 29, no. 7, pp. 1053-1060, 2003.

[30] M. P. Brewin, L. C. Pike, D. E. Rowland, and M. J. Birch, "The acoustic properties, centered on $20 \mathrm{MHZ}$, of an IEC agar-based tissue-mimicking material and its temperature, frequency and 
age dependence.," Ultrasound Med. Biol., vol. 34, no. 8, pp. 1292-306, Aug. 2008.

[31] L. M. Cannon, A. J. Fagan, and J. E. Browne, "Novel tissue mimicking materials for high frequency breast ultrasound phantoms.," Ultrasound Med. Biol., vol. 37, no. 1, pp. 122-35, Jan. 2011.

[32] S. T. Ostrowski and P. Tothill, "Kidney depth measurements using a double isotope technique," Br. J. Radiol., vol. 48, no. 568, pp. 291-294, Apr. 1975.

[33] D. Katritsis, L. Kaiktsis, A. Chaniotis, J. Pantos, E. P. Efstathopoulos, and V. Marmarelis, "Wall Shear Stress: Theoretical Considerations and Methods of Measurement," Prog. Cardiovasc. Dis., vol. 49, no. 5, pp. 307-329, 2007.

[34] T. G. Brott et al., "Stenting versus Endarterectomy for Treatment of Carotid-Artery Stenosis," N. Engl. J. Med., vol. 363, no. 1, pp. 11-23, Jul. 2010.

[35] M. Tanter and M. Fink, "Ultrafast imaging in biomedical ultrasound," IEEE Trans. Ultrason. Ferroelectr. Freq. Control, vol. 61, no. 1, pp. 102-119, Jan. 2014.

[36] J. Bercoff, "Ultrafast Ultrasound Imaging," in Ultrasound Imaging - Medical Applications, InTech, 2011.

[37] J. E. Browne, D. King, A. J. Fagan, D. Chari, and C. M. Moran, "An investigation of the detection capability of pulsed wave duplex Doppler of low grade stenosis using ultrasound contrast agent microbubbles - An in-vitro study," Ultrasonics, vol. 96, pp. 48-54, Jul. 2019.

[38] D. M. King, C. M. Moran, M. Hussey, and J. E. Browne, "How Fat Layers Affect the Clinical Diagnosis from Doppler Data," in 2007 IEEE Ultrasonics Symposium Proceedings, 2007, pp. 2457-2460.

[39] S. Kefayati and T. L. Poepping, "Transitional flow analysis in the carotid artery bifurcation by proper orthogonal decomposition and particle image velocimetry," Med. Eng. Phys., vol. 35, no. 7, pp. 898-909, Jul. 2013.

[40] M. Kadbi et al., "4D UTE flow: A phase-contrast MRI technique for assessment and visualization of stenotic flows," Magn. Reson. Med., vol. 73, no. 3, pp. 939-950, Mar. 2015.

[41] S. E. Lee, S.-W. Lee, P. F. Fischer, H. S. Bassiouny, and F. Loth, "Direct numerical simulation of transitional flow in a stenosed carotid bifurcation," J. Biomech., vol. 41, no. 11, pp. 25512561, Aug. 2008.

[42] B. Y. S. Yiu, A. J. Y. Chee, G. Tang, W. Luo, and A. C. H. Yu, "High frame rate doppler ultrasound bandwidth imaging for flow instability mapping," Med. Phys., vol. 46, no. 4, pp. 1620-1633, Apr. 2019. 